\title{
System Level Performance Evaluation of LTE with MIMO and Relays in Reuse-1 IMT-Advanced Scenarios
}

\author{
Rainer Schoenen ${ }^{1}$, Carlos Teijeiro and Daniel Bültmann, \\ Communication Networks (ComNets), Faculty 6, RWTH Aachen University \\ rs@comnets.rwth-aachen.de
}

\begin{abstract}
Cellular radio systems of the next generation will have to comply with the IMT-Advanced guidelines. Advanced radio techniques must be integrated into wireless systems to achieve the high spectral efficiency numbers required. Multiple antenna techniques such as MIMO are beneficial in regions of high SINR and low spatial stream correlation. Multihop techniques with fixed relays increase the throughput in regions close to the cell edge where SINR is typically low. Another challenge is the reuse-1 paradigm which worsens the interference situation. In this paper a mathematical model on system level is proposed and applied which allows to calculate system performance measures such as spectral efficiency. The MIMO model allows fast system evaluation without a lot of parameters and is therefore suitable for MAC layer simulative evaluation. The paper compares performance results for these techniques.
\end{abstract}

Index Terms-IMT-Advanced, LTE, Relays, MIMO

\section{INTRODUCTION}

$\mathbf{T}$ HE demand for higher data rates forces engineers to aim for the highest spectral efficiency ever. The cellular architecture of $3 \mathrm{G}$ systems cannot match this demand, so advanced techniques are needed on OSI layers one and two.

Due to high pathloss and interference near the cell border the offered data rate is one order of magnitude lower than close to the base station (BS). Even worse is that the same data rate occupies ten times the resources when using the lowest PhyMode (cell border) instead of the highest PhyMode near the base station .

It has been found that a good solution is the deployment of Fixed Relay Stations (FRS), also called Relay Nodes (RN), that are connected over the same wireless technology. Such a Multihop architecture [1], [2] has the advantage of cheap and easy deployment, and serves like a BS for a cell area where capacity and coverage is increased.

In regions of high $S I N R$ multiple antenna techniques offer spectral efficiencies beyond the SISO Shannon bound. For this reason MIMO transmission will be a part of future standards. System evaluation, especially MAC layer analysis, suffers from very complicated channel models for MIMO, which have too many parameters to be generic and too high computational complexity for a full cell analysis. It is even unclear if these models capture the reality correctly enough, e.g. when it comes to MIMO interference, spatial and temporal correlation.

${ }^{1}$ Dr. Schoenen is currently at http://www.sce.carleton.ca/
In this paper a simplified physical layer (PHY) model for the MIMO transmission is proposed that allows to model the main effect, namely the higher bit capacity per resource block compared to a SISO transmission. With a spatial correlation parameter it is able to scale the MIMO gain depending on the scenario.

The next generation systems will realize very advanced and complex mechanisms [3] to achieve the demanded spectral efficiency. In this paper the Reuse-1 paradigm is analyzed without and with a basic fractional frequency reuse scheme.

Based on SINR, MI and PER calculations the capacity in each location point can be determined. For more basic scenarios this has been investigated in [4]. In several cellular scenarios defined by the ITU guidelines for the IMT-Advanced evaluation [5] the spectral efficiency was determined in this work. In [6] LTE-Advanced has been studied alternatively. The results show an important insight into the pros and cons of each proposal based on the LTE-Advanced key numbers.

The paper is organized as follows. The first section gives an overview of the simplified layer-1 (PHY) performance models used. The simplified system level (large scale) MIMO model is introduced next. Then the performance models for layer-2 (MAC) are given. The last section shows performance results in the SISO and MIMO plus the singlehop and multihop case. A concluding summary contains statements to the key contributions in this paper.

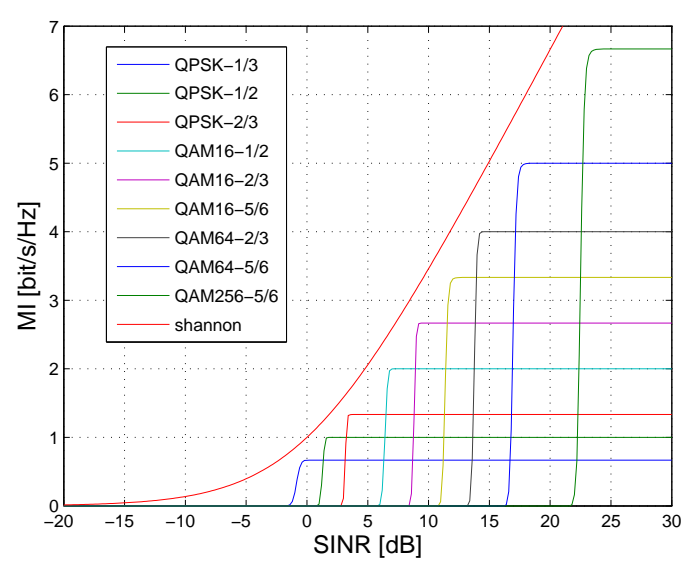

Fig. 1. Link level performance (net MI) for different modulation\&coding schemes (PhyModes). QAM256 is not used here. 
TABLE I

PHYMODES AND SINR INTERVALS

\begin{tabular}{|c|c|c|c|c|c|c|c|c|}
\hline SINR & 0.9 & 2.1 & 3.8 & 7.7 & 9.8 & 12.6 & 15.0 & 18.2 \\
\hline Mod. & \multicolumn{3}{|c|}{ QPSK } & \multicolumn{3}{c|}{ QAM16 } & \multicolumn{3}{c|}{ QAM64 } \\
\hline Cod. & $1 / 3$ & $1 / 2$ & $2 / 3$ & $1 / 2$ & $2 / 3$ & $5 / 6$ & $2 / 3$ & $5 / 6$ \\
\hline
\end{tabular}

\section{SIMPLIFIED PHY LAYER MODELS}

In order to determine higher layer system level results with reasonable time and effort, there must be an abstraction from the physics to mathematical models suitable of capturing all relevant effects. The techniques modeled and assumed in this paper are given here.

Adaptive modulation and coding (AMC) is a key feature of OFDM schedulers. The decision is based upon the $S I N R$ level expected at the receiver. It requires channel state information (CSI) which it can obtain by channel quality indication (CQI). Dynamic subchannel assignment (DSA) requires this information to choose the right subfrequency for each UT. In a MIMO capable system, each spatial stream has its own SINR performance which must be estimated and accounted for in all scheduling decisions. Section III explains the model assumed for MIMO channels.

From $S I N R$ to bit capacity ([bits/s/Hz]) this requires steps which are based on the mutual information (MI) method [7]. This works by applying the steps $S I N R \rightarrow M I$, $M I \rightarrow B E R$ and $B E R \rightarrow P E R$ to get the packet error probability. The PhyModes are determined by Tab. I. $M I$ has the meaning of the number of effective bits that can be transported at a certain $S I N R$ level. The following dependency is used [8]:

$M I(S I N R, m)=\frac{1}{\left(\left[s \cdot M I_{\text {shannon }}(S I N R)\right]^{-w}+m^{-w}\right)^{1 / w}}$

using the following abbreviations

$$
\begin{gathered}
M I_{\text {shannon }}(S I N R)=\log _{2}\left(1+10^{S I N R / 10 d B}\right) \\
s=s(m)=0.95-0.08 \cdot(m \bmod 2) \\
w=w(m)=2 \cdot m+1
\end{gathered}
$$

$m$ is the modulation index, i.e. the number of bits per symbol (1=QPSK,...8=QAM256). The $M I$ value has the unit of $[\mathrm{Mbit} / \mathrm{s} / \mathrm{Hz}]$, so we can derive the data rate by multiplying with the bandwidth of the subchannel. The net PHY throughput is obtained by multiplying with the coding rate. For LTE, coders have rate $1 / 3,1 / 2,2 / 3$ and $5 / 6$ [9].

The pathloss model captures the effects of the propagation power reduction. Here the IMT-Advanced evaluation standard models have been used. The detailed parameters depend on the scenario (Tab. II) and can be found in [5]. The main difference to the classical pathloss models is that there are two distinct values for either line-of-sight (LOS) or non-LOS (NLOS) and a probability function $p_{L O S}(d)$ to decide the likelihood of LOS or NLOS at a position in BS-UT distance $d$. An antenna downtilt has been accounted for (Tab. II).

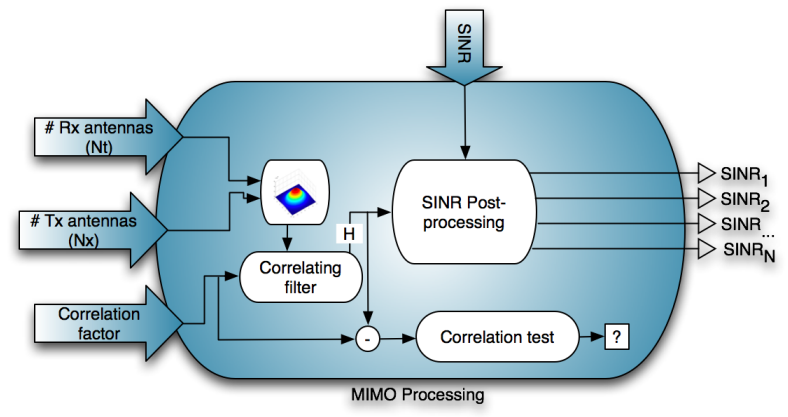

Fig. 2. Obtaining spatial SINR results for MIMO

\section{A MIMO MODEL FOR SYSTEM LEVEL EVALUATION}

In the past systems had single antennas only and it was sufficient to consider pathloss, antenna gain, fading, shadowing and interference to get the SINR value at the receiver. For MIMO using spatial layers, after receiver algorithms processed the received signal, the spatial layers $l$ each have their $S I N R_{l}$ which is proportional to the $S I N R_{S I S O}$ assumed for SISO. Due to physical effects it is limited, so that the $S I N R_{l}$ are usually lower than $S I N R_{S I S O}$ (see Fig. 3). Each spatial stream has its own PhyMode depending on $S I N R_{l}$ and the same mapping as in section II. A full channel information is assumed, although it may not be easy to get for MIMO. Due to $M$ spatial streams assumed, the performance of MIMO transmission goes beyond SISO when $M I_{\text {sum }}$ is big enough:

$$
M I_{\text {sum }}=\sum_{i=1}^{M} M I\left(S I N R_{l}\right)
$$

The calculation of the postprocessing $S I N R_{l}$ is done by a model according to [10], by which the post-processing $S I N R_{l}$ on stream $l$ is given by Eq. 6:

$$
S I N R_{l}=\frac{E}{M_{t} N_{0}} \frac{1}{\left[H^{H} H\right]_{l, l}^{-1}}
$$

where $H_{l, l}$ stands for the $(l, l)$ entry of the channel matrix $H$. The typical $l$ distributions of $S I N R_{l}$ are shown in Fig. 3.

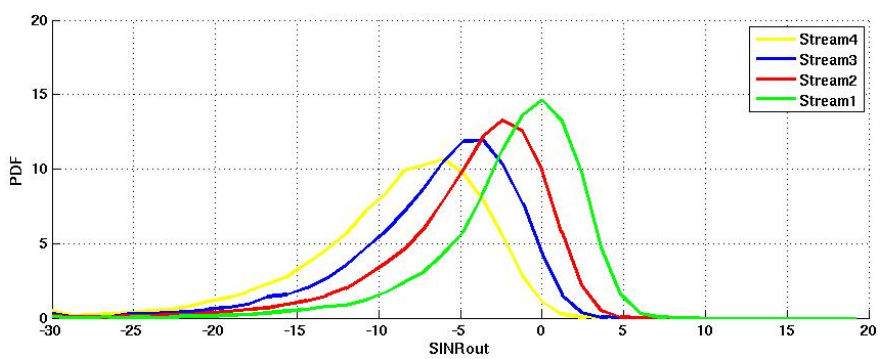

Fig. 3. PDF of post-processing $\triangle S I N R$ with MMSE receiver

Figure 2 shows the approach used here with just one parameter that characterizes the degree of independence of the spatial streams by a correlation factor $c$. This $c$ is used to construct a covariance matrix $R$. An eigenvalue decomposition of matrix $R$ is used to obtain the diagonal matrix $D$ and the corresponding eigenvectors in the columns of a matrix $V$ according to Eq. 7.

$$
\mathbf{R}=\mathbf{V}_{\mathbf{R}} \mathbf{D}_{\mathbf{R}} \mathbf{V}_{\mathbf{R}}{ }^{H}
$$


A correlating filter $\mathbf{W}$ can be formed with these eigenvalues:

$$
\mathbf{W}=\mathbf{V}_{\mathbf{R}} \sqrt{\mathbf{D}_{\mathbf{R}}}
$$

By applying the filter $\mathbf{W}$ to the totally uncorrelated channel $\mathbf{H}_{\mathbf{w}}$ we obtain the correlated channel matrix in Eq. 9 needed for Eq. 6.

$$
\mathbf{H}=\mathbf{W H}_{\mathbf{w}}
$$

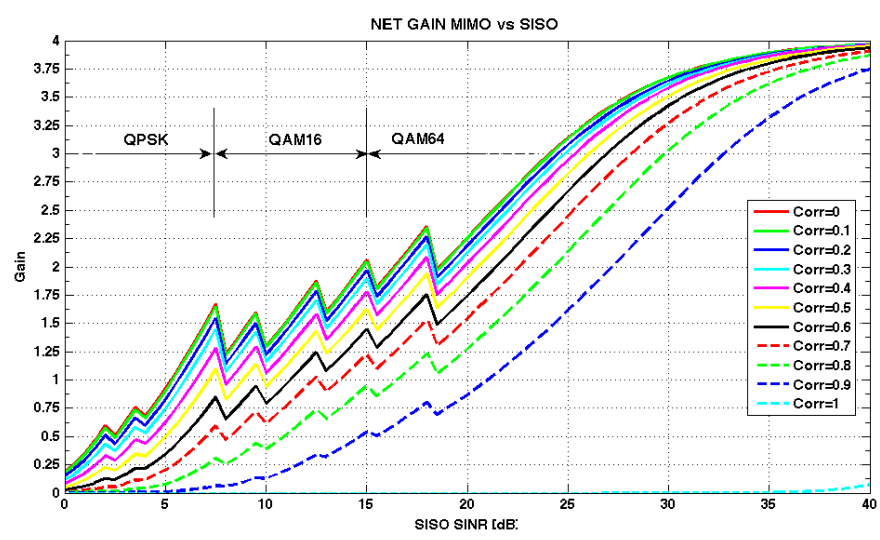

Fig. 4. Net gain between MIMO and SISO $M I$ in $[$ bits $/ s / H z]$ depending on antenna correlation parameter

The net MIMO gain shown in Fig. 4 is determined by dividing $M I_{\text {sum }}$ of Eq. 5 by the $M I_{S I S O}$. In this paper we assume single-user MIMO with $M=4 \mathrm{Tx}$ and Rx antennas. See [11] for more details on this model.

\section{MAC LAYER PERFORMANCE MODEL}

In the MAC layer a mapping must be performed to account for packet errors and ARQ retransmissions. The resulting net rate is given by $r_{\text {above } A R Q}=r_{\text {below } A R Q} \cdot(1-P E R)$. Also the MAC layer has overhead due to elements in the frame structure which are not related to net data transmission. Taking these effects into account, the result $r_{M A C}=f(S I N R)$ can be derived. Figure 1 shows this rate function for the 3G-LTE PhyModes.

From link level to MAC throughput, the performance of the example system is now evaluated by calculating the following steps. Parameters not found here are according to the IMTAdvanced evaluation guidelines [5]. The pathloss is explicitely modeled by two cases (LOS and NLOS) with a distance dependent probability $p_{L O S}(x, y)$ of having one or the other case [5]. All calculations accounted for this by determining two $S I N R$ levels, $S I N R_{L O S}$ and $S I N R_{N L O S}$ and average only at the end (Eq. 10), while the interference is averaged before (Eq. 11). See [6] for an alternative permutation model.

$$
\begin{aligned}
& M I(x, y)=M I_{L O S}(x, y) \cdot p_{L O S}+\left(1-p_{L O S}\right) \cdot M I_{N L O S}(x, y) \\
& I(x, y)=\sum_{\forall i} I_{L O S}^{(i)}(x, y) \cdot p_{L O S}^{(i)}+\left(1-p_{L O S}^{(i)}\right) \cdot I_{N L O S}^{(i)}(x, y)
\end{aligned}
$$

- Transmit Power $P_{T x}$ : see Table II,

- Pathloss: see Table II, Eq. 10 and [5],

- Interference I: neighbor cell BSs and neighbor sectors interfere ( $100 \%$ load, cluster order 1, Eq. 11)
TABLE II

IMT-ADVANCED SCENARIO SPECIFICATIONS

\begin{tabular}{|c|c|c|c|c|}
\hline Scenario & $\begin{array}{c}\text { Urban } \\
\text { micro } \\
\text { UMi }\end{array}$ & $\begin{array}{c}\text { Urban } \\
\text { macro } \\
\text { UMa }\end{array}$ & $\begin{array}{c}\text { Suburban } \\
\text { macro } \\
\text { SMa }\end{array}$ & $\begin{array}{c}\text { Rural } \\
\text { macro } \\
\text { RMa }\end{array}$ \\
\hline$d_{B S-B S}$ & $200 \mathrm{~m}$ & $500 \mathrm{~m}$ & $1299 \mathrm{~m}$ & $1732 \mathrm{~m}$ \\
\hline$h_{B S}$ & $10 \mathrm{~m}$ & $25 \mathrm{~m}$ & $35 \mathrm{~m}$ & $35 \mathrm{~m}$ \\
\hline$r_{\min }$ & $10 \mathrm{~m}$ & $25 \mathrm{~m}$ & $35 \mathrm{~m}$ & $35 \mathrm{~m}$ \\
\hline Ant. tilt $\phi_{t}$ & $-12^{\circ}$ & $-12^{\circ}$ & $-6^{\circ}$ & $-6^{\circ}$ \\
\hline$f_{C}[G H z]$ & 2.5 & 2.0 & 2.0 & 0.8 \\
\hline$P_{T x}$ & $44 \mathrm{dBm}$ & $49 \mathrm{dBm}$ & $49 \mathrm{dBm}$ & $49 \mathrm{dBm}$ \\
\hline
\end{tabular}

TABLE III

SECTORS AND ANTENNA DIRECTIVITY

\begin{tabular}{|c|c|c|}
\hline Sectors & 3 & 6 \\
\hline Antenna aperture horizontal $\theta_{3 d B}$ & $70^{\circ}$ & $35^{\circ}$ \\
Antenna aperture vertical $\phi_{3 d B}$ & $15^{\circ}$ & $15^{\circ}$ \\
\hline
\end{tabular}

- Noise $N$ : accounted for but not serious ( $I$-limited),

- SINR: $S I N R=S /(N+I)$,

- MI: mutual information determined from $S I N R$ and modulation (sec. II),

- BER: bit error ratio, depends on $M I$,

- PER: packet error ratio, the result after channel decoding,

- Throughput: determined by bandwidth, PhyMode (modulation and code rate), ARQ overhead,

- Spectral Efficiency: net $M I$ is throughput per bandwidth averaged over the cell (sector) area [8],

- Relays: least resources BS/RN association [8],

One example for the $P_{R x}$ and $P_{I f}$ in a typical scenario is shown in Fig. 5.

The MAC layer is also responsible for resource and packet scheduling [12]. In an uncoordinated frequency reuse (UFR), the assignment of OFDMA subchannels to users happens independently of the assignment in neighbor cells or sectors. In this paper we have investigated this and a simple coordinated resource allocation (CRA) in terms of a fractional frequency reuse (FFR) scheme applied near the sector borders. Because of a certain amount $a_{F F R}$ of resources (subchannels) not used in each sector, the spectral efficiency is noticeably reduced, which cancels out part of the gain due to FFR. The effect of FFR is the avoidance of inter-sector interference (Fig. 6(b)).

\section{A. Numerical Results}

Figure 6 shows results over the cell area for the IMTAdvanced scenarios defined in table II and [5]. The results use the analytic model of section II and III and did not use any simulation. The interference of neighbour cells and sectors with the same layout is properly taken into account with a frequency reuse of one (most dense package). The DL SINR results plotted over the cell area show the SINR of the best

TABLE IV

TECHNOLOGY PARAMETERS ACCORDING TO LTE-A

\begin{tabular}{|c|c|}
\hline Bandwidth [MHz] & FDD: 20DL,20UL \\
\hline Traffic & full load; best effort \\
\hline Antenna gain (boresight) & $17 d \mathrm{Bi}$ \\
\hline Thermal noise & $-174 d \mathrm{Bm} / \mathrm{Hz}$ \\
\hline UT noise figure & $5 d B$ \\
\hline
\end{tabular}




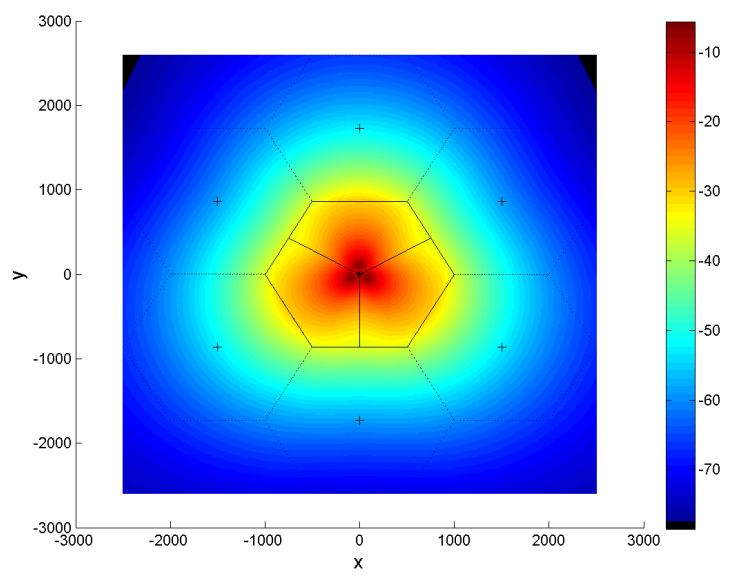

(a) RxPower/dBm with 3 sectors and directional antennas

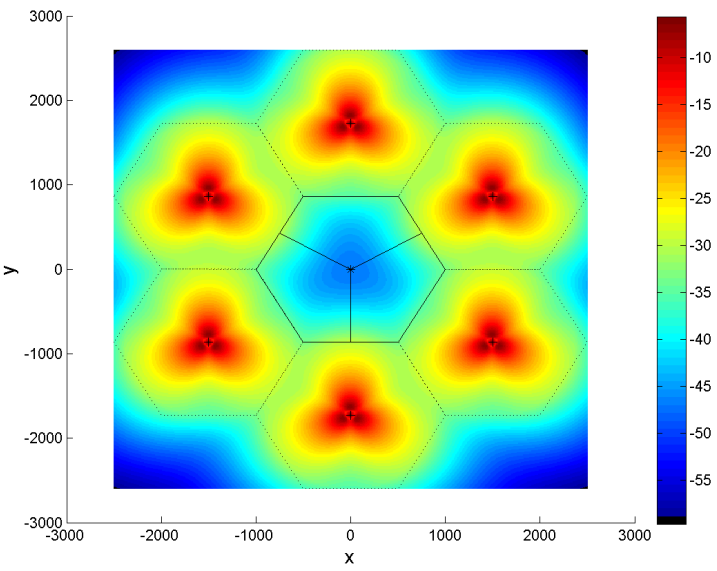

(b) Interference Power $(\mathrm{dBm})$ of the scenario left

Fig. 5. Example Scenario (RMa) of the IMT-Advanced evaluation. Interference assumed worst case (all subchannels used)

TABLE V

SPECTRAL EFFICIENCY RESULTS FOR THE SCENARIO EVALUATION $[b i t / s / H z /$ Sector $]$, GROSS WITHOUT MAC FRAME OVERHEAD

\begin{tabular}{|c|c|c|c|c|}
\hline Scenario & UMi & UMa & SMa & RMa \\
\hline 3S,UFR,0RN & 1.567 & 1.254 & 1.234 & 1.974 \\
\hline 3S,UFR,3RN & 1.945 & 1.804 & 1.825 & 2.310 \\
\hline 3S,UFR,6RN & 1.690 & 1.920 & 2.031 & 2.003 \\
\hline 3S,FFR,0RN & 1.980 & 1.649 & 2.010 & 2.522 \\
\hline 3S,MIMO-0.0,UFR,0RN & 3.971 & 2.871 & 2.853 & 5.740 \\
\hline 3S,MIMO-0.8,UFR,0RN & 3.525 & 2.875 & 2.906 & 5.009 \\
\hline 6S,UFR,0RN & 1.336 & 1.184 & 1.160 & 1.626 \\
\hline 6S,UFR,6RN & 1.581 & 1.927 & 1.996 & 1.781 \\
\hline 6S,FFR,0RN & 1.961 & 1.714 & 2.079 & 2.557 \\
\hline 6S,MIMO-0.0,UFR,0RN & 2.780 & 2.453 & 2.564 & 3.762 \\
\hline
\end{tabular}

station (BS,RN), not the maximum $S I N R$. Relays (3 or 6 if used) are assumed on half the distance to the cell border. The rate/throughput results contain the maximum achievable rate at a certain position within the cell, taking also the required first hop resources into account. The relay is chosen as the serving station (association) if this is an advantage (i.e. less resources used), which is here the same as having the maximum rate.

Table $\mathrm{V}$ shows the spectral efficiency results for the analyzed scenarios. $3 \mathrm{~S}$ vs $6 \mathrm{~S}$ means 3 or 6 sectors, respectively. UFR,FFR,MIMO- $c$ mean plain uncoordinated, fractional frequency reuse or $M I M O-c$ with cochannel correlation $c$. ORN,3RN,6RN mean the number of relays employed.

The results show that both Relays and MIMO give a performance benefit, even if the cochannel correlation is $c=80 \%$. But MIMO operates only well in areas of rather high $S I N R$, while relays are best located in areas of otherwise low $S I N R$. A combination of FFR and MIMO is also advised, but not taken here. In a real system there must be a combined solution that selects MIMO where the SINR and channel correlation is suitable and otherwise switches back to SISO with receive diversity combining.

\section{CONCLUSiON}

This paper presented a modeling framework to obtain performance results for IMT-Advanced scenarios including tech- niques such as MIMO and fixed relays. The method was then applied to singlehop and multihop cellular scenarios where all calculations were done analytically/numerically using Matlab.

The obtained results show the remarkable differences when using one or the other technology and give quantitative numbers for the spectral efficiency in those cases.

Future work will include a more detailed LOS/NLOS distinction for all possible combinations of interferers [6] and a MIMO model with distance dependent correlation properties.

\section{REFERENCES}

[1] R. Pabst, B. Walke, D. C. Schultz, H. Yanikomeroglu, and et al, "Relay-Based Deployment Concepts for Wireless and Mobile Broadband Radio," IEEE Communications Magazine, pp. 80-89, Sep 2004.

[2] M. Salem, A. Adinoyi, M. Rahman, H. Yanikomeroglu, D. Falconer, Y.-D. Kim, E. Kim, and Y.-C. Cheong, "An overview of radio resource management in relay-enhanced OFDMA-based networks," IEEE Communications Surveys and Tutorials, vol. 12, no. 3, Q3 2010.

[3] J. Monserrat, P. Sroka, G. Auer, J. Cabrejas, D. Martin, A. Mihovska, R. Rossi, A. Saul, and R. Schoenen, "Advanced Radio Resource Management for IMT-Advanced in WINNER+ (II)," in Proceedings of the ICT-MobileSummit 2010, Florence, Italy, Jun 2010, p. 9.

[4] R. Schoenen, W. Zirwas, and B. Walke, "Capacity and Coverage Analysis of a 3GPP-LTE Deployment Scenario," in Proceedings of the BWAW'08 colocated with ICC'08, Beijing, China, May 2008, p. 6.

[5] ITU, "Report ITU-R M2135; Guidelines for evaluation of radio interference technologies for IMT-Adcanced", 2008.

[6] D. Bültmann, T. Andre, and R. Schoenen, "Analysis of 3GPP LTEAdvanced Cell Spectral Efficiency," in Proc. of the PIMRC'2010, Istanbul, Turkey, September 2010, p. 6.

[7] K. Brueninghaus and D. e. a. Astely, "Link performance models for system level simulations of broadband radio access systems," in Proceedings of the 17th PIMRC'2005, Sep 2005, pp. 2306-2311.

[8] R. Schoenen and B. Walke, "On PHY and MAC performance of 3GLTE in a multi-hop cellular environment," in Proceedings of the IEEE WiCom'2007, Shanghai, China, Sep 2007, p. 6.

[9] K. J. e. a. Ekstrom H., Furuskar A., "Technical solutions for the 3G long-term evolution," IEEE Communications Magazine, Mar 2006.

[10] A. Paulraj, R. Nabar, and D. Gore, Introduction to space-time wireless communications. Cambridge: Cambridge University Press, 2003.

[11] R. Schoenen and C. Teijeiro, "A correlation-based MIMO Performance Model for the System Level Analysis on MAC layer," in submitted to the 12th intl' Conference on Communications Systems, Singapore, November 2010, p. 6.

[12] R. Schoenen and F. Qin, "A Closed Loop Control Model separating Resource and Packet Scheduling in Multihop Cellular Networks," in Proc. of the European Wireless, Lucca, Italy, Apr 2010, p. 6. 


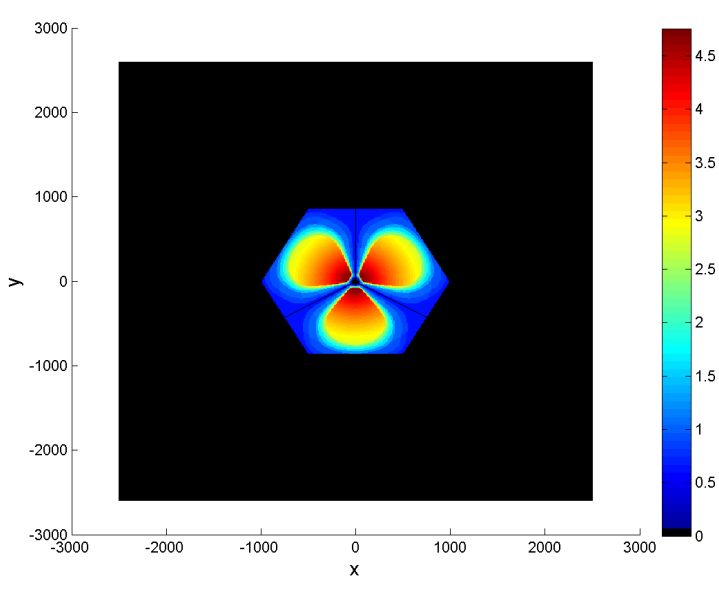

(a) RMa scenario, UFR, MI/(bit/s/Hz)

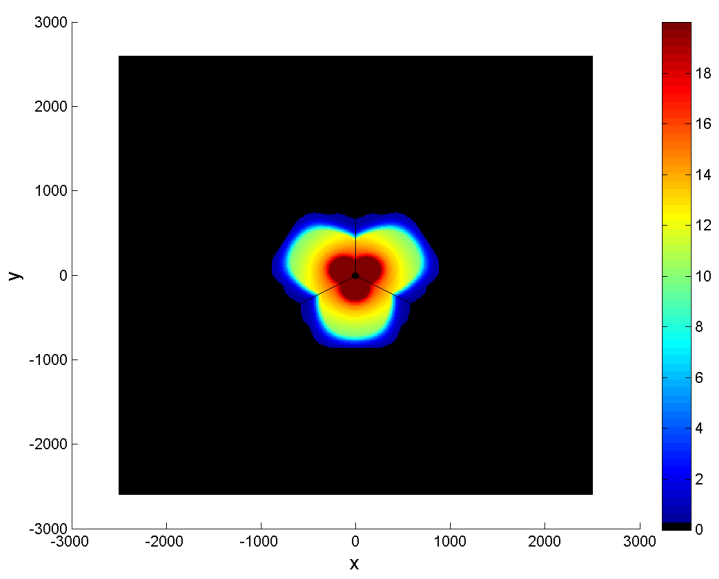

(c) RMa scenario with FFR+MIMO MI/(bit/s/Hz)

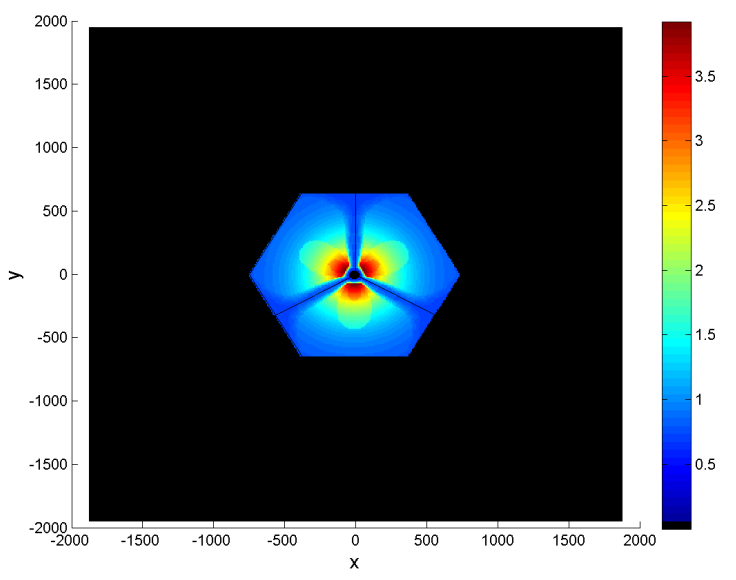

(e) SMa scenario MI/(bit/s/Hz)

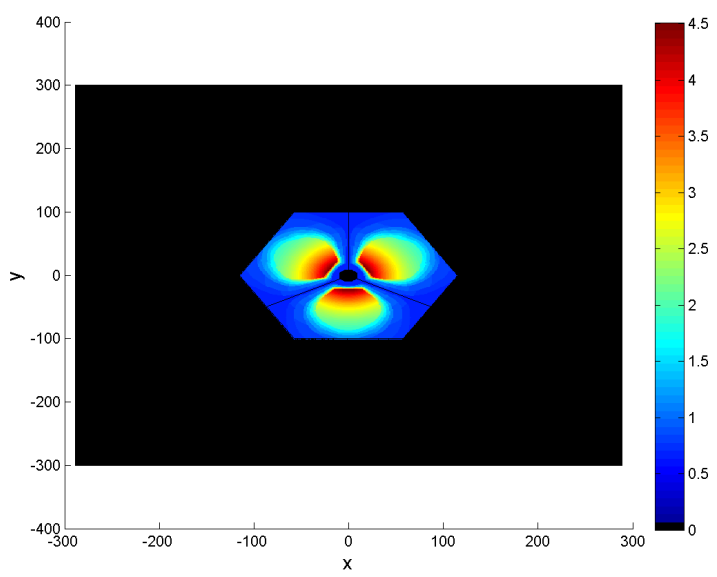

(g) UMi scenario $\mathrm{MI} /(\mathrm{bit} / \mathrm{s} / \mathrm{Hz})$

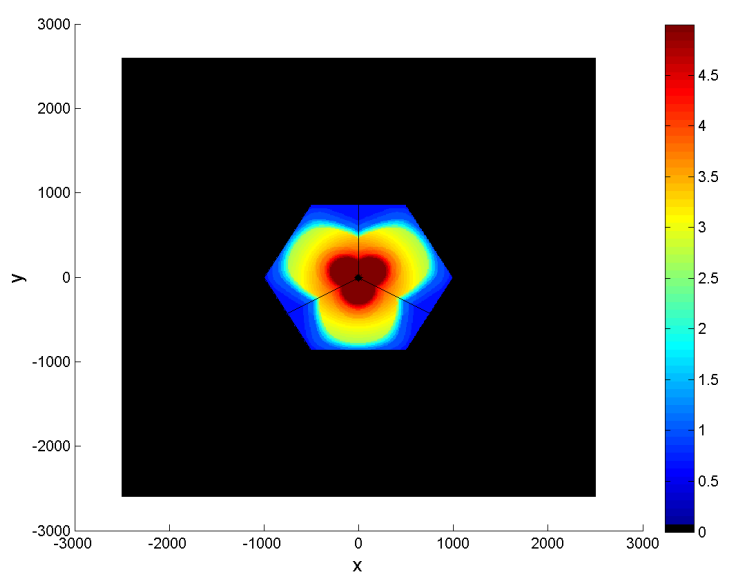

(b) RMa scenario with FFR MI/(bit/s/Hz)

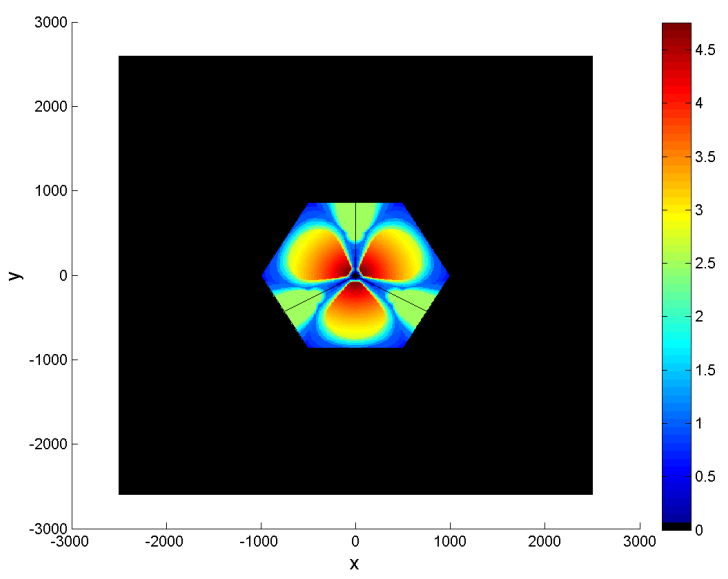

(d) RMa scenario with Relays MI/(bit/s/Hz)

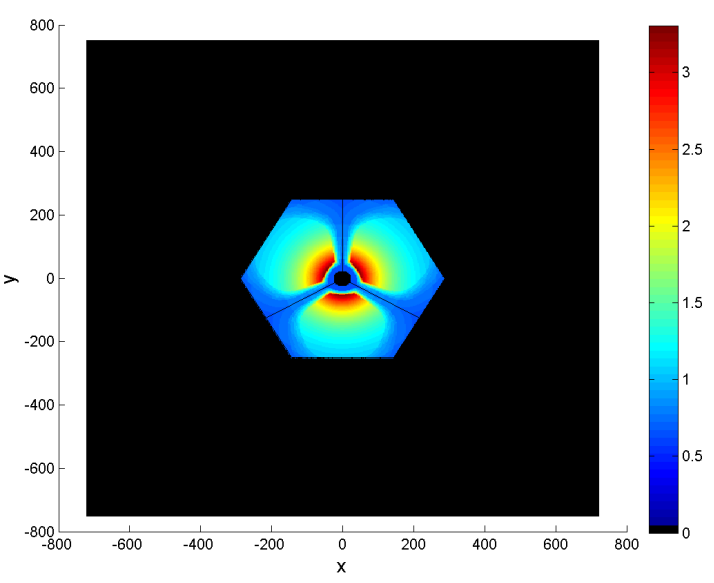

(f) UMa scenario $\mathrm{MI} /(\mathrm{bit} / \mathrm{s} / \mathrm{Hz})$

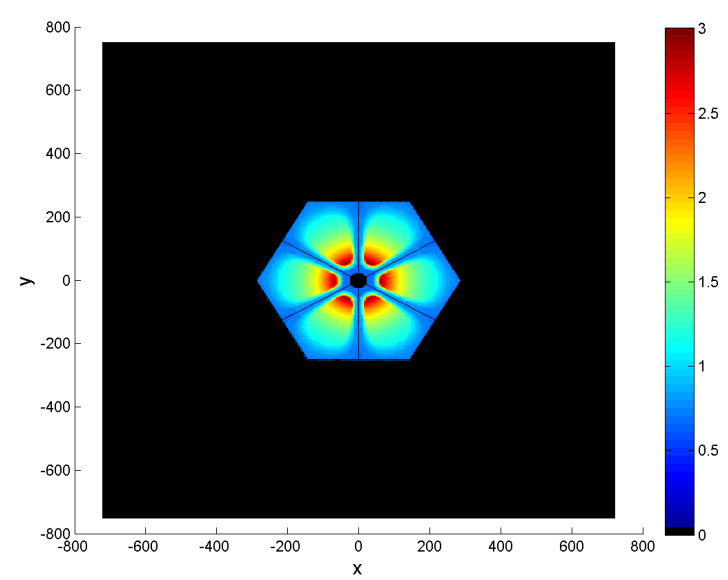

(h) UMa scenario $\mathrm{MI} /(\mathrm{bit} / \mathrm{s} / \mathrm{Hz}), 6$ sectors

Fig. 6. Area results for the different scenarios (IMT-Advanced) 